metallisch leiten und ausserdem $O$ zu lösen im stande sein. Ein Oxyd mit solchen Eigenschaften erlaubt aber die Methode nicht, von einem Edelmetall $z u$ unterscheiden. Zudem wird Eisen auch durch Cyankali passiviert, und diese Passivitat ist sicher weder durch Bildung einer Oxydschicht noch durch Sauerstoffbeladung zu erklären, da Cyankali reduzierend, nicht oxydierend wirkt. Die Messung des elektromotorischen Verhaltens von passivem Eisen gegen Eisenlösungen scheitert daran, dass passives Eisen, in eine Eisenlösung gebracht, sofort seine Passivität verliert, wenn kein Oxydationsmittel anwesend ist. Verf. beschränkt sich daher, ausgehend von der bekannten Thatsache, dass eine Elektrode aus gewöhnlichem Eisen gegen eine Ferri- stets negativer als gegen eine gleich konzentrierte Ferrosalzlösung ist, darauf, die Abhängigkeit der elektromotorischen Kraft des gewöhnlichen Eisens vom Oxydationszustand der Eisenlösung $z u$ untersuchen, und findet, dass die in das stärker oxydierte Gemisch von $F \ddot{*}$ - und $F e$ Salz tauchende Elektrode nicht allein stets die negativere ist, sondern dass auch ihr Potential andauernd sinkt, während sie sich gleichzeitig auflöst. Es hatte den Anschein, als ob sie die in ihre Nähe gelangenden Schichten der Lösung reduzierte und dabei selbst edler wurde. Eisen lässt sich also in Ferrilösung passivieren. Aus den Messungen scheint hervorzugehen, dass die Passivität das Endglied der Zustände ist, die es annimmt, wenn man in der umgebenden Lösung das Konzentrationsverhältnis $\frac{F_{\ell} \cdots}{F_{\ell} *}$ bis zu hohen Werten dieses Quotienten steigert, wonach dann die Passivität mit einer Oxydbildung nichts zu thun haben würde. - Den Verlauf kathodischer Zersetzungskurven (von Ferrilösungen), die ausgeprägte Knickpunkte aufweisen, erklärt Verf. so, dass sich Eisen in einer edeln - passiven Modifikation ausscheidet und ausserordentlich schnell in die aktive ubergeht, die sich in der Ferrilösung unter Reduktion auflöst. Allerdings konnte diese Abscheidung weder an Gold-noch an Platinelektroden sichtbar beobachtet werden. Dagegen scheinen die anodischen Zersetzungskurven von Ferrolösungen ein wirklicher Beweis dafür zu sein, dass Eisen nicht durch eine Oxydschicht passiviert wird. Denn das Bildungspotential passiven Eisens (in 0,2- und 2,0-norm. $\mathrm{H}_{2} \mathrm{SO}_{4}$ sowie in schwefelsaurer $0,2-$ und $\mathrm{I}, \mathrm{o}-$ norm. $\mathrm{Fe}_{2}\left(\mathrm{SO}_{4}\right)_{3}$-Lösung) war unabhängig von der Konzentration der Wasserstoffionen, dagegen abhängig von der Konzentration der $F e$-Ionen $(0,02$ Volt für die Zehnerpotenz des Eisengehalts). Zum Schlusse stellt Verf. eine Hypothese über die Natur des passiven Eisens auf, wonach dieses dreiwertiges Eisen in metallischem Zustand ist, und kann damit die meisten Resultate seiner Arbeit gut deuten. Bei der Erklärung anderer Thatsachen aber lässt die Hypothese vollständig im Stich.

\title{
BUNSEN - GESELLSCHAFT?
}

Es ist zweifellos richtig, dass die Ziele unserer Gesellschaft in Hinblick auf deren Entwicklung in den letzten Jahren erweiterungsfähig und - bedürftig sind, ja man kann behaupten, dass, falls wir nicht auf das gesamte Gebiet der physikalischen Chemie Hand legen, über kurz oder lang ein anderer Verein mit entsprechenden Zielen entstehen kann und muss.

Es ist darum nicht nur eine einfache Forderung weiser Vorsicht, sondern sogar das Gebot zwingender Notwendigkeit, dass wir unsere Statuten im Einklang mit unseren neuen Zielen ändern und dadurch der Entstehung eines Konkurrenzvereines, der ja doch nur eine uns allen unerwünschte Zersplitterung der Kräfte bedeuten würde, im Keime ersticken.

Was Herr Professor Ostwald hierüber und hierfür im allgemeinen in seiner vorjährigen Rede auf $\mathrm{Bunsen}$ erörterte, und Herr Professor Bredig im Anschluss daran itn einzelnen noch weiter ausführte, darin sind sich beinahe alle Mitglieder der Gesellschaft, soweit sie wenigstens bisher das Wort ergriffen haben, einig. Nicht aber in einem anderen Punkte, dem ich im Gegensatze zu einer bisher geäusserten Meinung durchaus nicht eine so geringe Bedeutung zusprechen möchte. Das ist nämlich die Wahl des Namens. Der Name einer Gesellschaft erscheint mir nach aussenhin als ihr Programm. Wer in eine Gesellschaft eintreten will, hört zuerst nur ihren Namen, und wird erst nach seinem Eintritt mit ihren Statuten und Einrichtungen wäher bekannt, so dass kaum daran zu zweifeln ist, dass der Name einer Gesellschaft den Inhait ihrer Statuten und Einrichtungen präcisieren muss.

In unserem Falle unterliegt nun die Wahl des Namens, wie sich bisher gezeigt hat, ganz besonderen Schwierigkeiten. Das darf uns aber durchaus nicht abschrecken, die Wahl eines Namens nach bestem Könneu vorzunehmen, da wir sonst eventuell Gefahr liefen, die Erweiterung der Ziele unserer Gesellschaft ganz fallen lassen zu müssen.

Erinnere man sich doch der Schwierigkeiten, die bei Gründung des Deutschen Reiches die Titulatur des Primus der deutschen Fürsten ob, , Kaiser von Deutschland " oder "Deutscher Kaiser" gemacht hat, Man hat damals doch den richtigen Namen im Interesse der Sache finden können!

Es ist bisher nur ein Name in Vorschlag gebracht worden, das ist die Anregung, die Deutsche Elektrochemische Gesellschaft zum Andenken an den grossen Namen Bunsen in "Deutsche Bunsengesellschaft" umzutaufen. 
Die Gründe, die Herr Professor Ostwald ursprünglich and in Erwiderung von Einwänden dargelegt hat, finden, wie ich gestehen muss, voll und ganz meine Billigung, und ich würde mich mit einer Verwirklichung jenes Planes durchaus befreunden können. Ich glaube aber denuoch, dass diese Verwirklichung daran scheitern kann, dass ein Name gewählt würde, der nicht direkt aus der Sache herausgewachsen ist.

Ich wüsste einen Vorschlag, der diesen Bedenken gerecht wird, ohne dass man darauf zu verzichten brauchte, in Ehrung eines grossen Namens die neuen Ziele unserer Gesellschaft in dem alten Namen mitzubezeichnen. Es wäre der Ausweg, den alten Namen und den neuen Vorschlag miteinander zu verbinden und die Gesellschaft „Deutsche Elektrochemische Bunsen-Gesellschaft " zu nennen. Diese giebt dann das Vereinsorgan, „Dle Zeltschrift für angewandte physikalische Chemie", heraus.

Ich glaube, durch diese Aenderungen würde den Anschaunngen aller Parteien Rechnung getragen, insbesondere aber würde der Narne „Bunsen" in eine gewisse organische Verbindung mit der „Deutschen Elektrochemischen Gesellschaft" gebracht. Nachdem Bunsen nicht nur Physikochemiker, sondern auch präparativer Elektrochemiker - ich erinnere nur an die elektrolytische Darstellung des Chroms und Magnesiums - ferner auch der Schöpfer jener galvanischen Zelle war, die zu den Zeiten, wo es noch keine Dynamomaschine gab, die einzige Quelle starker Ströme bildete, so nehme ich an, dass auch der ,praktische Techniker" nichts dagegen einzuwenden hat, wenn unser Altmeister immer in Zusammenhang mit der Elektrochemie genannt wird. Auch die von Herrn Professor Abegg für den neuen Zeitschriftentitel geforderten Eigenschaften der Prägnanz, der zutreffenden Bezeichnung des Inhalts, der Kontinuität mit der Vergangenheit dürften durch obige Amendements berücksichtigt sein.

Allerdings verhehle ich mir nicht, dass auch gegen diese Benennung schliesslich noch Einwände möglich sind. Es liegt in der Zusammensetzung der Namen die Eigentümlichkeit, dass die durch den Namen Bunsen bezeichnete Erweiternng unserer Ziele durch das Attribut "Elektrochemisch" wieder im alten Sinne beschränkt wirkt, und sodann hätte es eben wenig Sinn, dass das Wort Bunsen aberhaupt in Aufnahme gebracht wird. Und falls diese Namenbildung nur als eine Ueberleitung zu einer späteren Benennung „Bunsen-Gesellschaft" dienen sollte, möchte mancher sagen, dass man diese Zwischenstufe überhaupt unterlassen sollte.
Sollte man sich wirklich mit keinem dieser Vorschläge befreunden können, dann wäre es vielleicht am zweckmässigsten, gerade im Hinblick auf Herrn Professor Ostwalds jüngste Aeusserungen (diese Zeitschrift 8 , Io, S. I35 linke Spalte, Zeile ro von oben), "dass es bei dem Antrage unverhältnismässig viel mehr auf die Sache als auf den Namen ankommt", eine ganz andere, die erweiterten Ziele allerdings vollständig deckende Benennung in Erwägutig zu ziehen. Ich meine nämlich, der Deutschen Elektrochemischen Gesellschaft den Namen "Deutsche physikalischchemische Gesellschaft" zu geben. Gegen diesen Namen liesse sich, wie mir scheint, sachlich gar nichts einwenden, und praktisch sicherlich viel weniger als gegen den Namen „Deutsche BunsenGesellschaft". Er deckt sich vollständig mit den touen Zielen der Gesellschaft und ist nach aussenhin ein neutraler, jedem leicht verständlicher Ausdruck. Mit dem Namen ,Deutsche Bunsen-Gesellschaft" verbindet er höchstens den Nachteil, dass er, wie diese, die Verbindung mit der Vergangenheit aufgiebt. Die Zeitschrift würde, falls man sie nicht "Zeitschrift für angewandte physikalische Chemie“ benennen wollte, den Titel „Berichte der Deutschen physikalisch-chemischen Gesellschaft "führen können.

Ich habe mir erlaubt, meine Gedanken deshalb zum Ausdruck zu bringen, weil ich bemerkt zu haben glaubte, dass man diese Frage trotz des von Herm Professor Abegg in Vorjahre gestellten Schlussantrages noch nicht in genügendem Umfange diskutiert hat. Es würde mich, und gewiss noch viele andere Mitglieder freuen, wenn die von Herrn Professor Ostwald angeregte Frage, welche unbestreitbar für die Gesellschaft von vitalstem Interesse ist, von dem Plenum auf der diesjährigen Versammlung it einer zweckmässigen Weise verbeschieden würde.

Ich möchte noch gestehen, dass mir von allen Lösungen als die beste ein radikaler Schritt erscheinen würde; wie er in der letzten Form ausgedrückt ist. Selbstverständlich begrüsse ich aber jede Lösung, welche eine Erweiterung der Ziele unserer Gesellschaft nach der physikalisch-chemischen Seite hin bedeutet.

Dr. Max Buchner,

Vorstand der elektrochemischen Abteilung

von C. F. Böhringer \& Söhne, Mannheim-Waldhof.

Berichtigung. In dem Briefe von $\mathrm{Haber,} \mathrm{S.} \mathrm{207,}$ Zeile 7 vor Schluss muss es statt "organisch - präparativ " heissen: ", anorganisch-präparativ".

\section{PREISAUFGABE DER TECHNISCHEN HOCHSCHULE BERLIN FÜR DIE ABTEILUNG FÜR CHEMIE UND HÜTTENKUNDE.}

Das in einer der deutschen Patentschriften

r. O. Lindemann . . . D. R. - P. Nr. $81640 / 1894$,

2. O. Frank . . . . . " " $95720 / 1895$,

3. Siemens \& Halske. . , " " 88202/18g6,

4. C. Höpfner . . . . , " " IOI 177/1895

vorgeschlagene Verfahren zur Erzeugung von Zink auf elektrolytischem Wege ist in kleinerem Maassstabe durch Laboratoriumsversuche nachzuprüfen und soweit als angängig, auf seine Bedeutung für die Zinkgewinnung im Grossbetriebe zu untersuchen. Es bleibt dem Bewerber unbenommen, nach Auswahl nicht nur eines, sondern auch mehrere der genannten Verfahren zu 